\title{
EDITORIAL
}

\section{The pathophysiology of high-lethality suicide attempts: a vital area of suicide research}

\author{
Leo Sher ${ }^{1,2}$ iD \\ ${ }^{1}$ James J. Peters Veterans' Administration Medical Center, Bronx, NY, USA. ${ }^{2}$ Icahn School of Medicine at Mount Sinai, New York, NY, USA.
}

Suicide research and prevention are critical public health tasks, especially nowadays, during the stressful and continuing coronavirus disease 2019 (COVID-19) crisis. ${ }^{1-3}$ High-lethality suicide attempters who behaviorally closely resemble suicide victims may also resemble them biochemically. ${ }^{4}$ Studies of the neurobiology of highlethality suicide attempts may shed light on the pathophysiology of suicide. Moreover, such studies may help find biological markers of suicide and identify targets for therapeutic drugs to reduce suicidality. Finding biological predictors of highly lethal suicide attempts may be of significant value in predicting suicide.

Some studies have been conducted on the pathophysiology of highly lethal suicide attempts. Recently, Aguglia et al. ${ }^{5}$ examined the complete blood count values in highand low-lethality suicide attempters and non-attempters. Individuals with a history of high-lethality suicide attempts had a higher number of neutrophils, mean platelet volume, neutrophil-lymphocyte ratio, and platelet-lymphocyte ratio, as well as a lower number of lymphocytes relative to both those with low-lethality suicide attempts and the control group. ${ }^{5}$ The same research group previously observed that lower total cholesterol and higher c-reactive protein serum levels were associated with high-lethality suicide attempts. ${ }^{6}$

We compared high and low-lethality drug-free depressed suicide attempters with comorbid alcoholism. ${ }^{7}$ The Beck Lethality Scale was used to measure the degree of medical damage caused by each suicide attempt. ${ }^{8}$ The scale was scored from 0 to $8(0=$ no medical damage, $8=$ death), with different anchor points for various suicide attempt methods. Patients with medical lethality scores $\geqslant 4$ were classified as high lethality suicide attempters, while those with medical lethality scores $<4$ were regarded as low-lethality attempters. The classification between low-lethality and high-lethality attempters was based on lifetime history. We found that cerebrospinal fluid 5-hydroxyindoleacetic acid (5-HIAA) levels were lower in high-lethality attempters than low-lethality attempters. Other studies have also indicated that reduced serotonergic activity is associated with more lethal suicide attempts. ${ }^{4,9,10}$ Malone et al. ${ }^{4}$ observed that patients with a history of a higher lethality suicide attempt had a significantly lower prolactin response

Correspondence: Leo Sher, James J. Peters Veterans' Administration Medical Center, 130 West Kingsbridge Road, Bronx, NY 10468, USA.

E-mail: Leo.Sher@mssm.edu

Submitted Sep 30 2021, accepted Nov 18 2021, Epub Feb 282022. to fenfluramine, even when controlling for cortisol, age, sex, weight, comorbid cluster B personality disorder, pharmacokinetic and menstrual cycle effects.

We also examined the relation between cerebrospinal fluid 5-HIAA, homovanillic acid, and 3-methoxy-4-hydroxyphenylglycol (MHPG) levels and maximum lethality of suicide attempts in patients with bipolar disorder at baseline and during a 2-year follow up. ${ }^{11}$ The Beck Lethality Scale was used to assess the lethality of suicide attempts. ${ }^{8}$ 5-HIAA, homovanillic acid, and MHPG levels negatively correlated with the maximum lethality of suicide attempts during the 2-year follow-up period. ${ }^{11}$ Our finding was the first observation that cerebrospinal fluid monoamine metabolite levels may be predictors of the lethality of suicide attempts in patients with bipolar disorder.

Alcohol use has been implicated as a risk factor for high-lethality suicide attempts. ${ }^{12,13}$ Alcohol may be used to enable more medically serious attempts because it can alter an individual's mood, control, and judgment, and therefore allows one to cause more serious self-injury. Our study showed that acute alcohol use increases the lethality of suicide attempts in individuals with mood disorders. ${ }^{13}$ This demonstrates the importance of interventions to decrease acute and chronic alcohol use in individuals with mood disorders and/or suicidal tendencies.

Medically serious suicide attempts that carry a significant risk of death are more frequent in older adults than among younger people. ${ }^{14}$ This probably reflects a larger degree of planning and lower doubt about dying by suicide among older people. ${ }^{1,14}$ Bereavement, illness, and disability probably contribute to suicide risk among older adults. Older people who attempt suicide often display cognitive impairments, particularly poor cognitive control. ${ }^{14,15}$ For example, a research group examined cognitive inhibition in older high-lethality suicide attempters who closely resembled suicide victims. ${ }^{15}$ The authors concluded that poor cognitive control in high-lethality suicide attempters may weaken their ability to resolve real-life difficulties, triggering a disastrous accumulation of stressors. Preventing highly lethal suicide attempts among geriatric patients is probably a cornerstone of suicide prevention in older adults.

How to cite this article: Sher L. The pathophysiology of highlethality suicide attempts: a vital area of suicide research. Braz J Psychiatry. 2022;44:237-238. http://dx.doi.org/10.1590/1516-44462021-2281 
Identifying biological and psychological risk factors and attempt characteristics that contribute to high-lethality suicidal behavior has important clinical implications. Results from previous studies may be used to refine intervention efforts aimed at preventing high-lethality suicidal behavior or to treat individuals who have made medically serious attempts. Studies of the pathophysiology of high-lethality suicide attempts are an incredibly important but possibly underappreciated area of suicide research.

\section{Disclosure}

The author reports no conflicts of interest.

\section{References}

1 De Leo D, Padoani W, Scocco P, Lie D, Bille-Brahe U, Arensman E, et al. Attempted and completed suicide in older subjects: results from the WHO/EURO Multicentre Study of Suicidal Behaviour. Int J Geriatr Psychiatry. 2001;16:300-10.

2 Rihmer Z, Kántor Z, Rihmer A, Seregi K. Suicide prevention strategies--a brief review. Neuropsychopharmacol Hung. 2004;6:195-9.

3 Sher L. Suicide research and prevention during and after the COVID19 pandemic. Acta Psychiatr Scand. 2020;142:353-4.

4 Malone KM, Corbitt EM, Li S, Mann JJ. Prolactin response to fenfluramine and suicide attempt lethality in major depression. $\mathrm{Br} J$ Psychiatry. 1996;168:324-9.

5 Aguglia A, Amerio A, Asaro P, Caprino M, Conigliaro C, Giacomini G, et al. High-lethality of suicide attempts associated with platelet to lymphocyte ratio and mean platelet volume in psychiatric inpatient setting. World J Biol Psychiatry. 2021;22:119-27.
6 Aguglia A, Solano P, Giacomini G, Caprino M, Conigliaro C, Romano $\mathrm{M}$, et al. The association between dyslipidemia and lethality of suicide attempts: a case-control study. Front Psychiatry. 2019;10:70.

7 Sher L, Oquendo MA, Grunebaum MF, Burke AK, Huang YY, Mann JJ. CSF monoamine metabolites and lethality of suicide attempts in depressed patients with alcohol dependence. Eur Neuropsychopharmacol. 2007;17:12-5.

8 Beck AT, Beck R, Kovacs M. Classification of suicidal behaviors: I. Quantifying intent and medical lethality. Am J Psychiatry. 1975:132 285-7.

9 Nordström P, Samuelsson M, Asberg M, Träskman-Bendz L, Aberg-Wistedt A, Nordin C, et al. CSF 5-HIAA predicts suicide risk after attempted suicide. Suicide Life Threat Behav. 1994;24:1-9.

10 Samuelsson M, Jokinen J, Nordström AL, Nordström P. CSF 5-HIAA, suicide intent and hopelessness in the prediction of early suicide in male high-risk suicide attempters. Acta Psychiatr Scand. 2006;113: 44-7.

11 Sher L, Carballo JJ, Grunebaum MF, Burke AK, Zalsman G, Huang $Y Y$, et al. A prospective study of the association of cerebrospinal fluid monoamine metabolite levels with lethality of suicide attempts in patients with bipolar disorder. Bipolar Disord. 2006;8:543-50.

12 Chesin MS, Jeglic EL, Stanley B. Pathways to high-lethality suicide attempts in individuals with borderline personality disorder. Arch Suicide Res. 2010;14:342-62.

13 Sher L, Oquendo MA, Richardson-Vejlgaard R, Makhija NM, Posner K, Mann JJ, et al. Effect of acute alcohol use on the lethality of suicide attempts in patients with mood disorders. J Psychiatr Res. 2009;43:901-5

14 Szanto K, Clark L, Hallquist M, Vanyukov P, Crockett M, Dombrovski AY. The cost of social punishment and high-lethality suicide attempts in the second half of life. Psychol Aging. 2014;29:84-94.

15 Richard-Devantoy S, Szanto K, Butters MA, Kalkus J, Dombrovski AY. Cognitive inhibition in older high-lethality suicide attempters. Int $\mathrm{J}$ Geriatr Psychiatry. 2015;30:274-83. 